\title{
New regulations on X-ray use: likely implications for dental practices
}

\author{
Professor Keith Horner, University of Manchester, Co-editor of FGDP(UK)'s \\ Selection criteria for dental radiography, has reviewed the draft lonising \\ Radiation Regulations 2017 and draft lonising Radiation (Medical Exposure) \\ Regulations 2018 and what they mean for dental practices.
}

New regulations relating to the use of $\mathrm{X}$-rays will come into force in early 2018. The Ionising Radiation Regulations 2017 ('IRR17') will take effect on 1 January 2018 and the Ionising Radiation (Medical Exposure) Regulations 2018 ('IRMER18') on 6 February 2018. They apply to England, Wales and Scotland. Separate regulations will be produced later for Northern Ireland.

At this stage, the regulations are still in draft format, but there are some changes that seem to be certain. In the past, a dental practice needed to notify the Health and Safety Executive (HSE), on a once-only basis, that it was using X-rays. Under IRR17, dental practices will be required to register with HSE using an online system (under development). It is understood that registration will require confirmation that certain actions have been taken, such as appointment of a Radiation Protection Adviser (RPA), risk assessments, adequate training of staff, amongst others. There will be a fee for registration which is understood to be at the 'tens of pounds' level. Re-registration will be required on a regular basis. Other changes under draft IRR17, which might have meant dental practices having to install additional barrier protection for staff, seem to have been successfully challenged.

Under IRMER18, dental practices will need to appoint a Medical Physics Expert. It is not expected that this will be a problem as the role will almost certainly combined with that of the RPA already required to be appointed. New procedures will be required relating to justification of exposures to 'carers and comforters' (eg a parent supporting a child undergoing an X-ray examination) and, if needed, for 'non-medical imaging' (eg exposures for medico-legal examinations). Other changes seem to have minimal impact on dentists.

You should expect your RPA to update you about the changes in the months ahead. Medical and dental guidance notes are planned for publication around May 2018, unfortunately after the regulations have come into force. There are no current plans to produce a specific dental guidance document. The FGDP(UK) will update their Selection criteria for dental radiography guidance to take account of the new regulations as soon as possible and may, if it is seen to be useful, develop some additional resources to help dentists understand the new regulations.

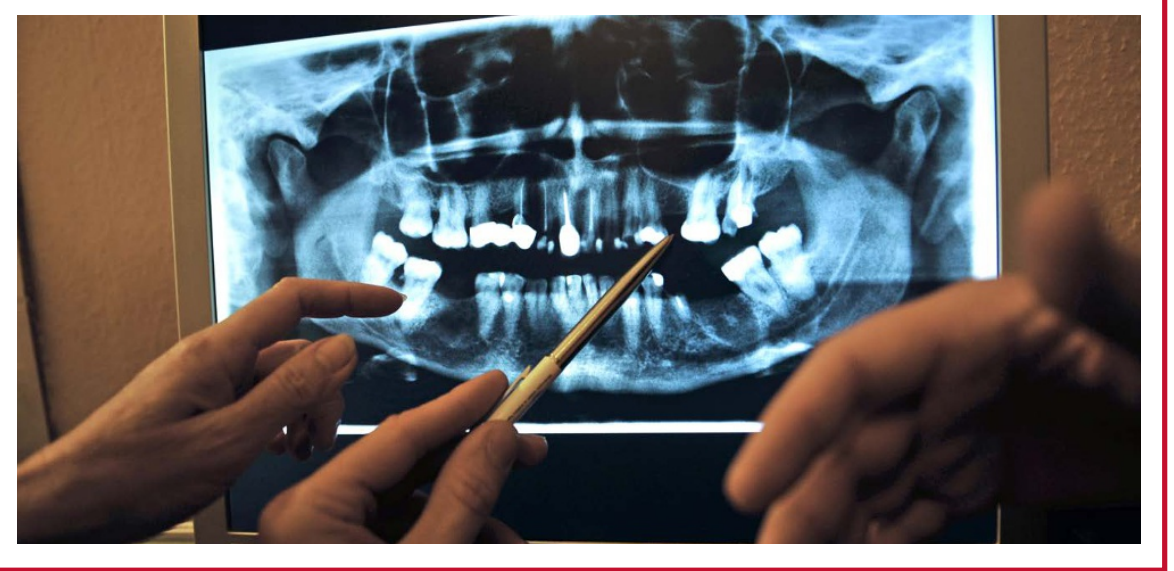

Africa Healthcare Summit 2018

The Africa Healthcare Summit, co-hosted with Africa Dental Summit 2018, will be held on 6-7 March 2018 at Olympia in London during the 5th Annual Africa Healthcare Week 2018. Pre-event roundtable discussions will be held at HMS Belfast in London on 5 March.

The Africa Healthcare Summit is the only event of its kind looking at current developments and opportunities within the African healthcare sector. The event has grown enormously over the last five years and has been extremely effective at bringing together top tier decision makers from the Ministries of Health, public and private hospitals and distribution chains to network and discuss past, present and future projects throughout Africa.

For further information visit https://www. africahealthcareweek.com/.

\section{Chewing gum developed that detects peri-implant disease}

A chewing gum that can detect periimplant disease was described in Nature Communications in August. ${ }^{1}$

Lorenz Meinel and colleagues developed a biosensor for detecting peri-implant disease that produces a bitter taste to indicate a positive diagnosis. They bound the sensor to another compound and embedded it into a chewing gum, which is tasteless in healthy patients. However, the occurrence of periimplant disease increases the production of specific enzymes that can cleave the biosensor and release the strong bitter compound. An 'artificial tongue' was used that can detect the bitter compound to distinguish between saliva samples from patients with peri-implant disease and asymptomatic volunteers.

Although the chewing gum has yet to be tested on volunteers in a clinical setting, it could remove the need for complex kits and expert intervention at the point of diagnosis. The authors suggest that in the future, the gum may be used in dental practices or for personal use by patients.

Ritzer J, Lühmann T, Rode C et al. Diagnosing peri-implant disease using the tongue as a 24/7 detector. Nat Commun 2017; 8: 264. DOI: 10.1038/s41467-017-00340-X. 